\title{
Negative mood-induction modulates default mode network resting-state functional connectivity in chronic depression
}

Citation for published version (APA):

Renner, F., Siep, N., Arntz, A., van de Ven, V., Peeters, F. P. M. L., Quaedflieg, C. W. E. M., \& Huibers, M. J. H. (2017). Negative mood-induction modulates default mode network resting-state functional connectivity in chronic depression. Journal of Affective Disorders, 208, 590-596. https://doi.org/10.1016/j.jad.2016.10.022

Document status and date:

Published: 15/01/2017

DOI:

10.1016/j.jad.2016.10.022

Document Version:

Publisher's PDF, also known as Version of record

\section{Document license:}

Taverne

Please check the document version of this publication:

- A submitted manuscript is the version of the article upon submission and before peer-review. There can be important differences between the submitted version and the official published version of record.

People interested in the research are advised to contact the author for the final version of the publication, or visit the DOI to the publisher's website.

- The final author version and the galley proof are versions of the publication after peer review.

- The final published version features the final layout of the paper including the volume, issue and page numbers.

Link to publication

\footnotetext{
General rights rights.

- You may freely distribute the URL identifying the publication in the public portal. please follow below link for the End User Agreement:

www.umlib.nl/taverne-license

Take down policy

If you believe that this document breaches copyright please contact us at:

repository@maastrichtuniversity.nl

providing details and we will investigate your claim.
}

Copyright and moral rights for the publications made accessible in the public portal are retained by the authors and/or other copyright owners and it is a condition of accessing publications that users recognise and abide by the legal requirements associated with these

- Users may download and print one copy of any publication from the public portal for the purpose of private study or research.

- You may not further distribute the material or use it for any profit-making activity or commercial gain

If the publication is distributed under the terms of Article $25 \mathrm{fa}$ of the Dutch Copyright Act, indicated by the "Taverne" license above, 
Research paper

\title{
Negative mood-induction modulates default mode network resting-state functional connectivity in chronic depression
}

\author{
Fritz Renner ${ }^{\mathrm{a}, \mathrm{b}, *, 1}$, Nicolette Siep ${ }^{\mathrm{b}}$, Arnoud Arntz ${ }^{\mathrm{c}}$, Vincent van de Ven ${ }^{\mathrm{d}}$, Frenk P.M.L. Peeters ${ }^{\mathrm{e}}$, \\ Conny W.E.M. Quaedflieg ${ }^{\mathrm{b}, \mathrm{f}}$, Marcus J.H. Huibers ${ }^{\mathrm{g}}$ \\ ${ }^{a}$ MRC Cognition and Brain Sciences Unit, Cambridge, United Kingdom \\ b Department of Clinical Psychological Science, Maastricht University, The Netherlands \\ c Department of Clinical Psychology, University of Amsterdam, The Netherlands \\ d Department of Cognitive Neuroscience, Maastricht University, The Netherlands \\ e Department of Psychiatry and Neuropsychology, University Hospital Maastricht, The Netherlands. School for Mental Health and Neuroscience, Faculty of \\ Health, Medicine and Life sciences, Maastricht University, The Netherlands \\ ${ }^{\mathrm{f}}$ Department of Cognitive Psychology, Institute for Psychology, University of Hamburg, Germany \\ ${ }^{\mathrm{g}}$ Department of Clinical Psychology, VU University Amsterdam, The Netherlands
}

\section{A R T I C L E I N F O}

\section{Keywords:}

Mood-induction

Chronic depression

Resting state fMRI

Default mode network

Posterior cingulate cortex

\begin{abstract}
A B S T R A C T
Background: The aim of this study was to investigate the effects of sad mood on default mode network (DMN) resting-state connectivity in persons with chronic major depressive disorder (cMDD).

Methods: Participants with a diagnosis of cMDD $(\mathrm{n}=18)$ and age, gender and education level matched participants without a diagnosis of depression $(n=18)$ underwent a resting-state fMRI scan, before and after a sad mood induction. The posterior cingulate cortex (PCC) was used as a seed for DMN functional connectivity across the two resting-state measurements.

Results: Mood ratings decreased in both groups following the sad mood induction procedure. PCC connectivity with the parahippocampal gyrus, the superior temporal gyrus and the anterior inferior temporal cortex increased in cMDD patients following the sad mood induction, whereas it decreased in non-patient controls. PCC connectivity with the anterior prefrontal cortex and the precuneus decreased in cMDD patients following the sad mood induction, whereas it increased in non-patient controls.

Limitations: Limitations of this study include the relatively small sample size and lack of a clinical control group.

Conclusions: These findings are in line with neurobiological models of depression suggesting that the observed changes in DMN connectivity following the sad mood induction might reflect a failure to exert cognitive control over negative memory retrieval in patients with cMDD.
\end{abstract}

\section{Introduction}

Major depressive disorder (MDD) is a relatively common, psychiatric disorder that often takes a chronic course [1]. Recent efforts to understand the neuropathology of depression have focused on connectivity within or between brain networks that can be identified during rest for reviews see e.g.: [2,3]. The default mode network (DMN), a brain network which has well-documented associations with self-referential processing [4], rumination [3,5] and autobiographic memory [6], plays a key role in the neuropathology of MDD [7]. Numerous studies have now demonstrated that patients with MDD show increased DMN resting-state functional connectivity, compared to non-patient controls $[2,8]$.

Although the finding of increased connectivity in the DMN of MDD patients is fairly consistent [2], connectivity within this network might not necessarily be stable. For example, a number of studies have shown that DMN connectivity in depression changes when symptoms improve following treatment with antidepressants [9]. Moreover, changes in mood states might also drive network dynamics. For example, studies in non-depressed [10] and remitted depressed [11] participants have shown that DMN connectivity can be modulated by a sad mood induction. Harrison et al. (2008) used a combination of autobiographical recall and classical music to induce a neutral (scan 1) or sad (scan 2) mood state in healthy participants. The authors found that the

\footnotetext{
* Corresponding author.

E-mail address: Fritz.Renner@mrc-cbu.cam.ac.uk (F. Renner)

${ }^{1}$ Postal address: MRC Cognition and Brain Sciences Unit; 15 Chaucer Road, Cambridge CB2 7EF, United Kingdom.
} 
strength of DMN connectivity decreased in the sad vs. neutral mood induction condition [10]. Zamoscik et al. (2014) used a combination of autobiographical recall and classical music to induce sad mood in individuals with remitted depression and healthy controls. The authors found that individuals with remitted depression showed greater connectivity between the PCC and parahippocampal gyri following the sad mood induction compared to healthy controls [11].

Whereas these previous studies reveal important mechanisms as to how DMN connectivity can be modulated by a sad mood induction in healthy or previously depressed individuals, it is unclear how patients with a current MDD modulate connectivity in the DMN following a sad mood induction. Given that connectivity within the DMN is positively correlated with depressive chronicity [12], it is intriguing to study DMN connectivity following a sad mood induction in patients with chronic major depressive disorder (cMDD). Therefore, the aim of the present study was to investigate resting state connectivity of the DMN in patients with a current cMDD and matched non-patient controls before and after a sad mood induction.

Harrison et al. (2008) instructed participants to actively visualize and ruminate about the event recalled. The authors argued that the sad mood-induction might require more cognitive effort from their healthy participants whereas neutral recall might require less cognitive effort. Given previous findings of task induced deactivation of the DMN they expected that the sad vs neutral mood induction would lead to reduced DMN connectivity [10]. However, DMN connectivity in individuals with depression, compared to non-depressed individuals, is generally increased and immersing in a sad mood state might be less effortful for individuals with depression compared to non-depressed individuals. Moreover, the sad-mood induction that was used in the current study was conducted prior to scanning and did not require participants to engage in a task during scanning. Finally, as has been argued by Zamoscik et al. (2014) a negative mood induction can trigger increased self-referential processing in (remitted) depressed patients, increasing DMN connectivity. Based on these previous findings it is hypothesized that compared to non-patient controls, cMDD patients would show increased connectivity in the DMN following a sad mood induction.

\section{Method}

\subsection{Participants}

Eighteen patients with chronic major depressive disorder (cMDD) and 18 age, education level and gender matched non-patient controls participated in this study. Non-patient controls were also matched on left/right handedness except for one left handed patient who could not be matched with a left handed control participant. Patients were recruited from a specialized secondary care outpatient unit in the Netherlands (Riagg Maastricht) as part of a treatment study [13]. All patients were scanned before the start of the intervention phase of the treatment study. Non-patient controls were recruited via poster advertisements and a volunteer database. Participants were excluded if they had MRI contraindications.

Inclusion criteria for patients in the treatment study were a current diagnosis of cMDD defined as meeting Diagnostic and Statistical Manual of Mental Disorders fourth edition (DSM-IV) criteria of major depressive disorder for $\geq 2$ years, assessed with the Structured Clinical Interview for axis-I Disorders (SCID-I) by trained interviewers and a Beck Depression Inventory second edition (BDI-II) score of 20 or higher at study entry. Exclusion criteria for patients in the treatment study were the presence of bipolar disorder, psychotic disorders, alcohol or substance dependence, autism spectrum disorders, clusterA or cluster-B personality disorder and acute suicide risk. One patient with co-morbid borderline personality disorder was not included in the treatment study but is included here. Use of antidepressant medication at study entry was an exclusion criterion, unless patients were stable on medications for three month or longer prior to study entry $(n=10$;
Table 1

Demographic and clinical characteristics of the sample.

\begin{tabular}{|c|c|c|c|c|}
\hline & $\begin{array}{l}\mathrm{cMDD}(N \\
=18)\end{array}$ & $\begin{array}{l}\text { Controls }(N \\
=18)\end{array}$ & $\begin{array}{l}t \text {-test ( } p \text { - } \\
\text { value) }\end{array}$ & $\begin{array}{l}\chi^{2}(p- \\
\text { value })\end{array}$ \\
\hline Age, M (SD) & $\begin{array}{l}41.17 \\
(17.53)\end{array}$ & $42.67(18.73)$ & 0.81 & - \\
\hline Gender, n (\%) & & & - & 1.00 \\
\hline female & $13(72.2)$ & $13(72.2)$ & & \\
\hline male & $5(27.8)$ & $5(27.8)$ & & \\
\hline Education level, n (\%) & & & - & 0.68 \\
\hline low & $1(5.6)$ & $2(11.1)$ & & \\
\hline medium & $6(33.3)$ & $4(22.2)$ & & \\
\hline high & $11(61.1)$ & $12(66.7)$ & & \\
\hline BDI-II score, M (SD) & $\begin{array}{l}30.50 \\
(9.39)\end{array}$ & $2.67(2.77)$ & $<0.001$ & - \\
\hline $\begin{array}{l}\text { \# Previous episodes, M } \\
\quad \text { (SD) }\end{array}$ & $3.38(2.39)$ & - & - & - \\
\hline $\begin{array}{l}\text { Antidepressant use at } \\
\text { baseline, n (\%) }\end{array}$ & $10(55.6 \%)$ & - & - & - \\
\hline
\end{tabular}

Note. BDI-II = Beck Depression Inventory second edition; $\mathrm{cMDD}=$ chronic Major Depressive Disorder. Information on number of previous episodes was available from 16 patients.

55.6\%). Control participants meeting any current DSM-IV axis-I disorder or lifetime mood disorder, as assessed with the SCID-I interview were excluded.

The study protocol was approved by the medical ethical committee of the academic community hospital Maastricht, the Netherlands (Protocol ID/number: NL 31871.068.10). After complete description of the study to the participants, written informed consent was obtained. The authors assert that all procedures contributing to this work comply with the ethical standards of the relevant national and institutional committees on human experimentation and with the Helsinki Declaration of 1975, as revised in 2008. Demographic and clinical characteristics of the sample are summarized in Table 1.

\subsection{Procedure}

This study used a mixed design with group (cMDD, non-patient controls) as between-subject factor and two fMRI runs (pre mood induction, post mood induction) as within subject factor. First, participants underwent a $6 \mathrm{~min}$ resting-state scan. Participants then rated their current mood along four visual analogue scales (VAS) ranging from 0 to 100 with higher scores representing more positive mood using an MR compatible joystick. The four VAS scales measured mood on a bipolar continuum between negative and positive, dull and glad, secure and anxious, and sad and happy. After the initial mood ratings, participants received the sad mood induction, consisting of mood suggestive music ("Adagio for Strings" by Samuel Barber played via headphones), played during the anatomical scan, in combination with autobiographical recall of a personal sad event ( $8 \mathrm{~min})$. This standard protocol reliably induces short lasting sad mood-states [14] and the specific piece of music has successfully been used in our previous studies $[15,16]$. Directly after the sad mood induction, current mood-state was again assessed along the four VAS scales. Following this, participants underwent the second resting-state fMRI scan (6 min). After the second resting-state scan participants again rated their current mood-state on the four VAS scales.

\subsection{Measures}

\subsubsection{Beck depression inventory}

Depressive symptom severity was assessed using the Beck Depression Inventory second edition (BDI-II), a 21-item self-report instrument [17]. In the present study the Dutch version of the BDI-II was used which has been shown to have high internal consistency in a Dutch sample of psychiatric patients (Cronbach's $\alpha=0.92$ ) and ade- 


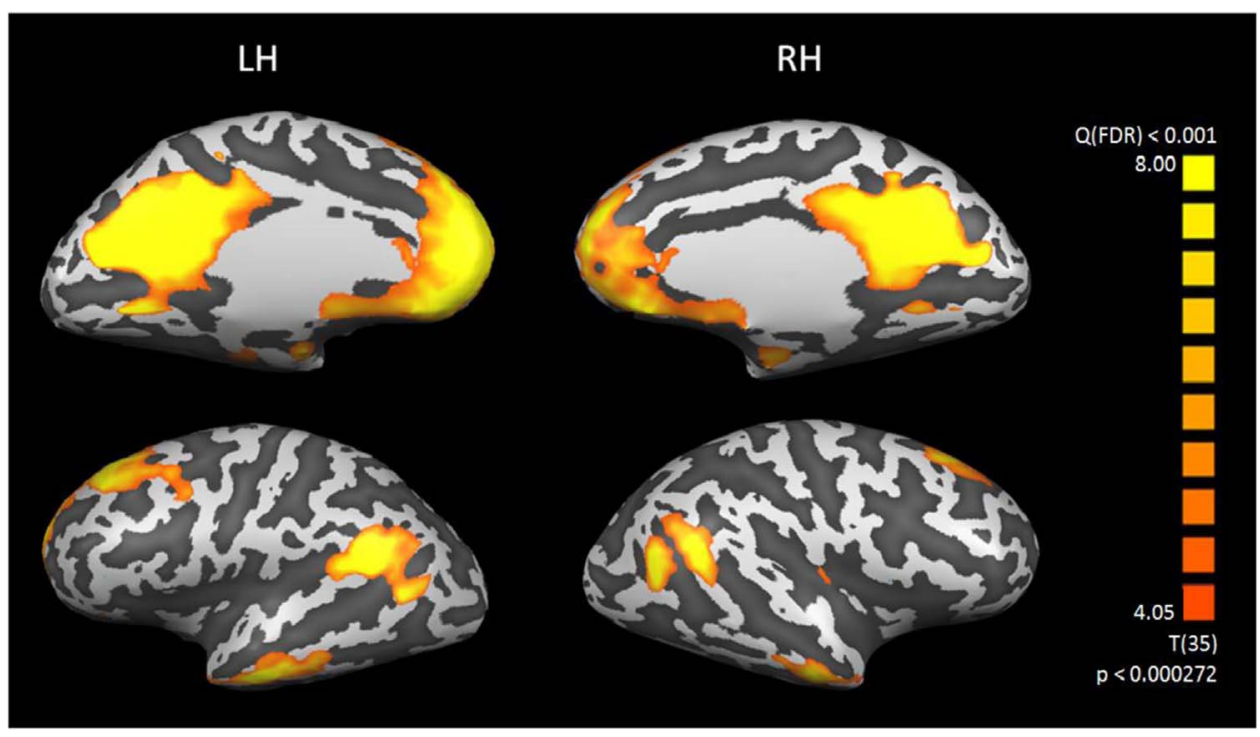

Fig. 1. PCC based functional connectivity mask resembling the default mode network..

quate construct validity with other depression scales rs between 0.79 and $0.85[18]$.

\subsection{Image acquisition}

Anatomical images were acquired using a Magnetom Allegra $3 \mathrm{~T}$ scanner (Siemens Healthcare, Netherlands) located at the Faculty of Psychology and Neuroscience, Maastricht University with a T1weighted gradient echo (196 slices, TR $=2250 \mathrm{~ms}$, TE $=26 \mathrm{~ms}$, flip angle $=90^{\circ}$, field of view $=256 \mathrm{~mm}$ and voxel dimensions $1 \times 1 \times 1 \mathrm{~mm}^{3}$ ). A T2*-weighted functional measurement was acquired using a standard echo-planar imaging (EPI) sequence (TR $2000 \mathrm{~ms}$, TE $30 \mathrm{~ms}$, flip angle $=90^{\circ}, 32$ slices, 180 volumes, $3 \times 3 \times 3 \mathrm{~mm}^{3}$ ). A slice tilt correction of -30 was used to minimize inhomogeneity artefacts [19].

\section{5. fMRI data preprocessing}

Data pre-processing and analyses were conducted with Brainvoyager QX v2.4 (Brain Innovations, Maastricht, the Netherlands). The first two volumes of the functional images were omitted due to magnetization artifacts. Preprocessing of the remaining functional data included slice time correction using sinc interpolation, 3D motion correction using sinc interpolation, spatial smoothing (Gaussian kernel with full-width-at-half-maximum of $4 \mathrm{~mm}$ ) and linear trend removal. Individual functional datasets were then co-registered with structural images of the same participant and subsequently normalized using the Talairach and Tournoux transformation procedure [20]. Talairach coordinates were subsequently transformed to Montreal Neurological Institute (MNI) coordinates based on [21]. Individual anatomical datasets were then averaged to create a groupbased anatomical brain mask to exclude voxels belonging to the outside of the brain.

\subsection{Resting-state functional connectivity analysis}

To determine change in DMN connectivity following the sad mood induction, we conducted a seed based connectivity analysis. The PCC was selected as seed because this region has been shown to reveal connectivity in the DMN most consistently [22]. The anatomical location of the PCC seed was based on the results of a separate selfreferential processing task, performed in the same experimental session by the same participants, following the resting-state scans [23]. In this task a whole-brain Random Effects (RFX) ANOVA analyses was used to contrast personality trait words with non-words. This analysis resulted in the functional localization of the cortical midline structures involved in self-referential processing [24], including the PCC. The peak voxel of the left PCC ( $5 \mathrm{~mm}$ sphere around MNI: $-9,-56,25)$ was selected as seed, which is comparable in location to regions that previous studies have used to reveal the DMN [11,25]. A $5 \mathrm{~mm}$ sphere has been previously used to define the PCC as seed area e.g. [26].

In order to perform the group seed based analysis in Brainvoyager QX, single study design matrix (SDM) files were created for each run of each participant using the NeuroElf MATLAB (Mathworks, Inc.) toolbox (www.neuroelf.net) and a resting state analysis toolbox developed at Maastricht University [27,28]. These SDM files included the BOLD time series of the PCC seed region and $122 \mathrm{z}$-normalized nuisance confound regressors: six 3D head motion parameters and their first derivatives, mean signal from the ventricles and white matter, global signal, and signal oscillations at a frequency above $0.1 \mathrm{~Hz}$ (sine-cosine pairs). The resulting SDM files were then used to run an RFX GLM to find voxels that showed correlation with the PCC seed for each run of each participant. The Pearson correlation coefficients $(r)$ maps were then transformed using Fisher's r-to-z transformation ( $\mathrm{z}=0.5 \mathrm{Ln}$ $[(1+r) /(1-r)])$, to yield variates that are approximately normally distributed. The resulting $F$-map showed all voxels that had a significant correlation with the PCC seed at a conservative false discovery rate (FDR) of 0.001 for all participants over all runs. This map was transformed into a DMN mask (Fig. 1), which was used to limit the final main effects and groups $\mathrm{x}$ mood induction analyses to the hypothesized DMN.

In the final analysis, first, the main effect of group on the first resting-state scan (pre mood induction) and the main effect of run (resting-state pre/post mood induction) was computed to replicate earlier findings of increased DMN connectivity in depressed patients and to determine the effect of the sad mood induction on DMN connectivity in general, respectively. Second, a repeated measures ANOVA analysis with group (non-patient control vs. cMDD) as between subject factor and mood induction (pre/post) as withinsubject factor was conducted to test for the hypothesized difference in the effects of the sad mood induction between the two groups. The resulting three $F$-maps were thresholded at a $p$-value of 0.01 and a cluster size threshold of $135 \mathrm{~mm}^{3}$ ( 5 voxels) as determined by the cluster-level statistical threshold estimator plugin (BrainVoyager QX; 1000 Monte Carlo simulations). 


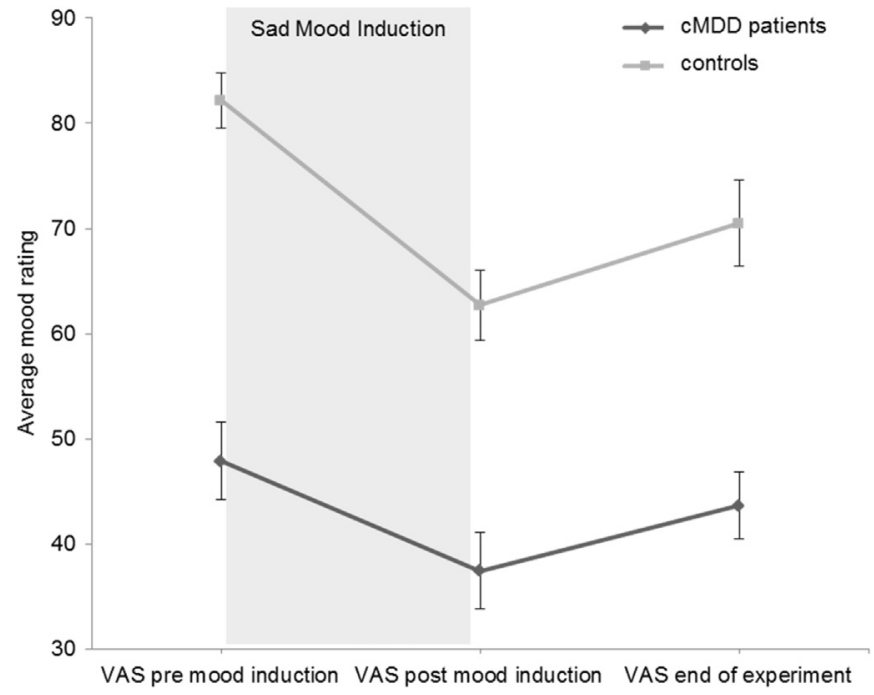

Fig. 2. Effects of the mood induction manipulation on self-reported mood ratings Mood ratings prior to the mood induction (T1), after the mood induction (T2) and at the end of the experiment (T3); higher scores represent more positive mood. Error bars represent standard error of the mean.

\section{Results}

\subsection{Behavioral data}

\subsubsection{Manipulation check}

Within group effects of the mood induction were assessed using paired $t$-tests and between group differences were assessed using repeated measures analysis of variance. To reduce statistical testing, an average mood scale of all four VAS scales was constructed per mood assessment moment (Cronbach's alpha $=0.91,0.88$, and 0.85). Fig. 2 shows average mood ratings before and after the sad mood induction and after the second resting state scan. Within group comparisons revealed a significant decrease in mood for non-patient controls and cMDD patients, $t(17)=6.04, p<0.001$ and $t(17)=3.18, p=0.005$, respectively), indicating the success of the sad mood induction procedure. The decrease in mood following the sad mood induction was slightly stronger in non-patient controls, but the interaction effect was not statistical significant $F(1,34)=3.87, p=0.057$. At the end of the experiment, mood state remained at a lower level compared to the pre mood induction assessment for both patients $(t(17)=2.76, p=0.01)$ and non-patient controls $(t(17)=3.46, p<0.01)$. At each time point there was a significant main effect of group, showing that patients reported a lower mood-state at pre mood induction, $t(34)=-7.58, p<0.001$, post
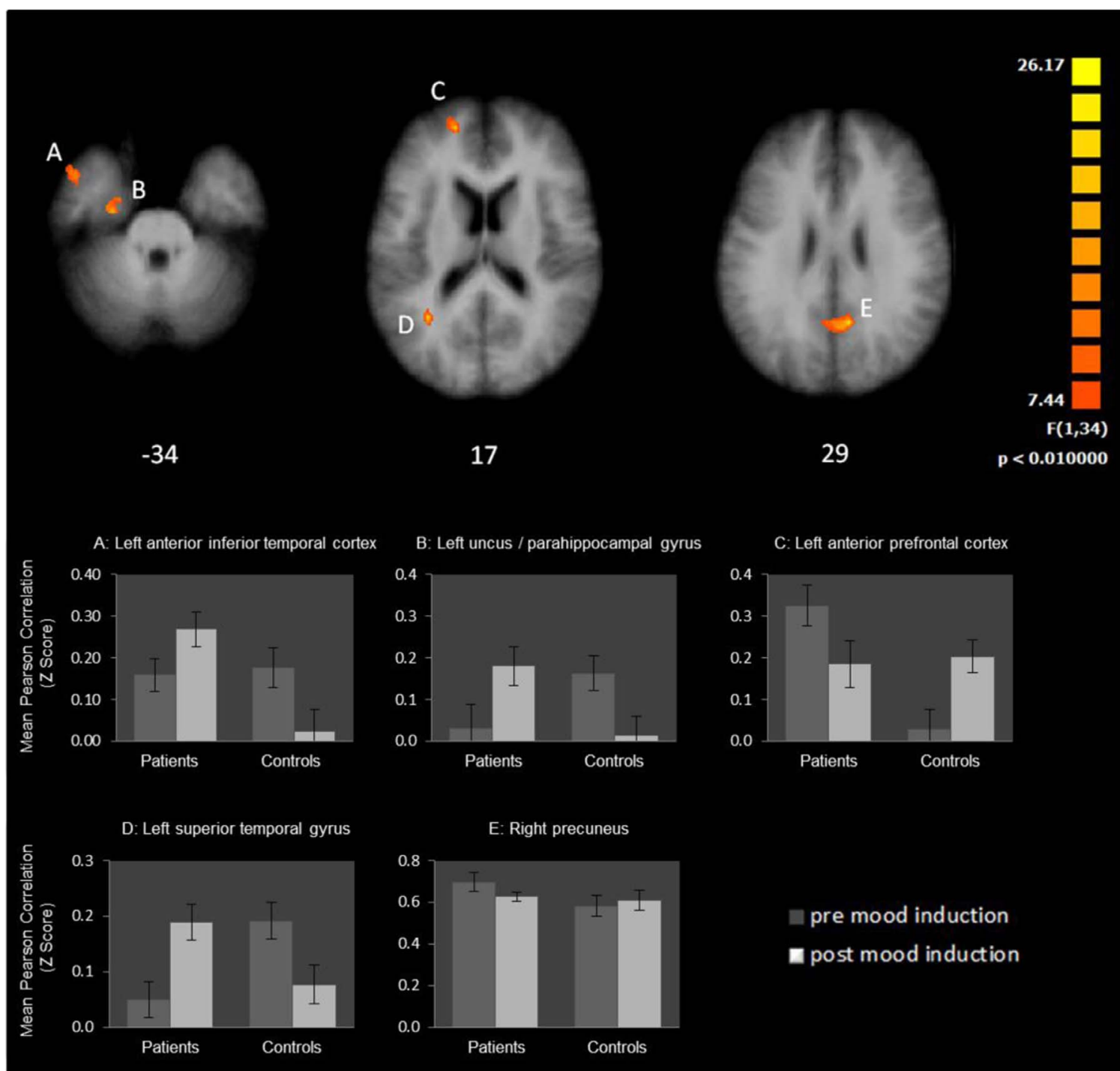

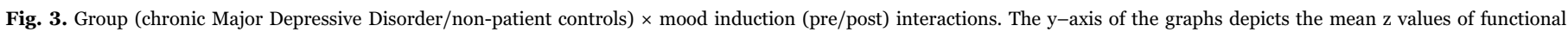
connectivity of the Posterior Cingulate Cortex with the Default Mode Network. Error bars represent standard error of the mean. 
mood induction, $t(34)=-5.11, p<0.001$, and after the second restingstate scan, $t(32)=-5.29, p<0.001$.

\section{2. fMRI results resting-state functional connectivity}

\subsubsection{Main effect group}

Prior to the sad mood induction cMDD patients, compared to nonpatient controls, showed greater connectivity between the PCC and the medial temporal gyrus $(52,-68,28)$, the dorsolateral medial prefrontal cortex $(10,61,30)$, the precuneus $(-6,-65,29)$, the anterior prefrontal cortex $(-6,57,30)$, the PCC $(-6,56,10)$, the superior occipital gyrus $(-45,-78,34)$ and the medial temporal gyrus $(-58,-59,15)$.

\subsubsection{Main effect pre to post mood induction}

There was an overall significant decrease in connectivity from pre mood induction to post mood induction in the precuneus $(-6,-58,35)$, and in two separate clusters of the parahippocampal gyrus $(-13,-33,-1$ and $-36,-25,-18)$.

\subsubsection{Group on mood induction interaction}

Five ROIs with significant group (cMDD/non-patient controls) $\times$ mood induction (pre/post) interactions were identified (Fig. 3; Table 2), including the anterior inferior temporal cortex, the superior temporal gyrus, the uncus/anterior parahippocampal gyrus, the anterior prefrontal cortex, and the precuneus. In line with our hypothesis the anterior inferior temporal cortex, the superior temporal gyrus and the uncus/anterior parahippocampal gyrus showed increased connectivity after the sad mood induction in patients with cMDD compared to non-patient controls. However, the precuneus and anterior prefrontal cortex showed the opposite pattern, with increased connectivity after the sad mood induction in non-patient controls compared to the patient group.

\section{Discussion}

The present study provides the first evidence, to our knowledge, that DMN connectivity in currently depressed participants is modulated by sad mood states. Using a PCC seed, main effect analyses of group prior to the sad mood induction replicated previous findings showing increased DMN connectivity in patients with depression, for a review see for example [29]. The DMN has been linked to selfreferential processing, rumination and autobiographic memory and therefore plays an important role in the neuropathology of depression. In this study we focused on connectivity within the DMN using the PCC as seed. Previous studies have also demonstrated increased cerebellarDMN connectivity in individuals with depression showing that subregions of the Cerebellum are linked to the DMN [30]. The main focus of the present study was on the hypothesized increase in DMN connectivity following the sad mood induction in cMDD patients. In line with this hypothesis, PCC connectivity with the anterior inferior temporal cortex, the superior temporal gyrus and the parahippocampal gyrus increased following the sad mood induction in cMDD patients. However, the precuneus, an important DMN node, showed a decrease in connectivity with the PCC in CMDD patients. Note that the simple effect of change in PCC-precuneus connectivity within the cMDD group was marginally significant $(\mathrm{p}=0.08)$. Non-patient controls showed an increased PCC connectivity with the anterior prefrontal cortex. These findings are in line with previous studies showing increased DMN connectivity in remitted depressed patients, compared to healthy controls, following a sad mood induction [11] and decreased DMN connectivity in healthy controls following a sad mood induction [10]. Together, empirical findings show that DMN connectivity in depressed patients can be modulated by subjective mood-states.

In general, the present finding of increased DMN connectivity, following the sad mood induction in cMDD patients supports neurobiological models of depression [31]. Importantly, in the current study the sad mood induction procedure was conducted before functional imaging of the second resting-state scan and no effortful cognitive processing was required by participants during the resting state scan. In contrast, non-clinical participants in Harrison et al. (2008) engaged in effortful processing during the resting state scan. Thus their finding of decreased connectivity following the sad vs neutral mood induction might be due to task-induced (sad mood induction) deactivation as the authors discuss [10]. A negative mood induction in (remitted) depressed patients might trigger self-referential processing and rumination, associated with increased DMN connectivity [11]. The increased connectivity following the sad mood induction in patients compared to non-patient controls in the current study therefore likely reflects a relatively natural reaction of patients, after being challenged with sad mood, rather than compliance of the task instruction to recall sad autobiographical events.

We found increased PCC - parahippocampal gyrus connectivity in patients with cMDD following the sad mood induction. Another study focusing on dynamic changes of functional connectivity within the DMN in individuals with depression used a MPFC seed and reported decreased dynamic connectivity with the parahippocampal gyrus potentially reflecting increased self-focus thinking [32]. In line with findings of the current study, another study [11] reported increased PCC - parahippocampal gyrus connectivity following a sad mood induction in previously depressed participants. The increased PCC parahippocampal gyrus connectivity in patients following the sad mood induction might reflect activation of negative thoughts and rumination in patients as has been suggested previously [11]. The results of the current study therefore provide further evidence for the idea that the parahippocampal gyrus plays a potentially important role in chronic depression [11].

The finding of decreased PCC - precuneus connectivity in patients versus controls following the mood induction was unexpected in the light of previous studies showing increased precuneus - DMN connectivity in patients with (chronic) depression [12]. The precuneus plays a key role in visual mental imagery, episodic memory recall and self-referential processing [33]. Thus, increased PCC - precuneus connectivity in non-patient controls in the present study might reflect episodic autobiographical memory retrieval triggered by the negative mood induction that has not been observed in previous studies not using mood-induction procedures. One previous study in medication

Table 2

Region of interest details identified for interaction between group (chronic Major Depressive Disorder vs non-patient controls) and pre and post-mood induction.

\begin{tabular}{|c|c|c|c|c|c|c|c|c|c|}
\hline \multirow[b]{2}{*}{ Brain region } & \multirow[b]{2}{*}{$\mathrm{L} / \mathrm{R}$} & \multirow[b]{2}{*}{ MNI (x,y,z) } & \multirow[b]{2}{*}{ Cluster size Voxels } & \multirow[b]{2}{*}{$\mathrm{F}$} & \multirow[b]{2}{*}{$p$-value } & \multicolumn{2}{|c|}{ Average connectivity change Fisher's Z } & \multicolumn{2}{|c|}{ Cohen's d (change within groups) } \\
\hline & & & & & & cMDD & controls & cMDD & controls \\
\hline Anterior inferior temporal cortex & $\mathrm{L}$ & $-49,9,-34$ & 262 & 13.83 & $<0.001$ & 0.11 & -0.15 & 0.37 & -0.46 \\
\hline Uncus / parahippocampal gyrus & $\mathrm{L}$ & $-26,-11,-28$ & 178 & 18.96 & $<0.001$ & 0.15 & -0.15 & 0.45 & -0.49 \\
\hline Anterior prefrontal cortex & $\mathrm{L}$ & $-16,52,18$ & 633 & 27.61 & $<0.001$ & -0.14 & 0.18 & -0.42 & 0.57 \\
\hline Superior temporal gyrus & $\mathrm{L}$ & $-32,-53,21$ & 184 & 28.98 & $<0.001$ & 0.14 & -0.11 & 0.53 & -0.43 \\
\hline Precuneus & $\mathrm{R}$ & $10,-52,31$ & 615 & 4.44 & 0.043 & -0.07 & 0.03 & -0.25 & 0.09 \\
\hline
\end{tabular}

Note. $\mathrm{L} / \mathrm{R}=$ Left / Right; Cohen's d = Mean difference/pooled SD. 
free patients with depression reported no differences in PCC precuneus connectivity between patients and controls [34]. In line with our finding of decreased PCC - precuneus connectivity in patients with CMDD, another study reported decrease PCC/precuneus and angular gyrus connectivity in patients with depression and this was associated with higher overgeneral autobiographical memory scores [35]. Overgeneral memory refers to a difficulty in retrieving specific memories a phenomenon that has been consistently associated with depression for a review see e.g. [36].

Patients with depression showed increased PCC - superior temporal gyrus and inferior temporal cortex connectivity following the sad mood induction compared to non-patient controls. The superior temporal gyrus plays a role in emotional processing and abnormalities in this region are consistently identified in Major Depressive Disorder [37]. Contrary to findings of the current study, previous studies generally found less activation of the superior temporal gyrus during processing of negative stimuli [38]. However, here we induced negative mood using a musical mood induction in combination with autobiographical memory recall and the superior temporal gyrus is also involved in music processing [39]. Interestingly, the superior temporal gyrus has also been linked to multisensory-emotional integration particularly for sad music [40]. The increased PCC - superior temporal gyrus connectivity in patients with depression following the sad mood induction in the current study might reflect a stronger integration of the sad music with sad autobiographical recall in patients with depression in the current study.

Non-patient controls uniquely increased PCC - anterior prefrontal cortex connectivity following the sad mood induction. Anterior prefrontal cortex subdivisions have previously been associated with cognitive control of memory retrieval $[41,42]$. Present findings might therefore be interpreted as an attempt of the non-patient controls to control retrieval of negative memories elicited by the sad mood induction. In line with this suggestion, patients with cMDD might fail to control retrieval of negative memories following the sad mood induction. This idea is in line with non-fMRI studies suggesting that depression is associated with impairments in cognitive control for a review see: [43] and with theoretical models proposing that reduced prefrontal control in combination with activation of negative schemas in depression gives rise to a downward spiral of rumination and negative affect [44]. Clinically, these findings suggest that patients with cMDD fail to control or stop negative memory retrieval once they are in a sad mood-state. In contrast, non-depressed individuals control memory retrieval during sad mood possibly reflecting a coping strategy that prevents healthy individuals from entering a negative downward spiral of dysfunctional cognitive processing and negative affect. Note that this explanation is based on theory and should be tested in future studies.

A number of limitations should be mentioned. First, we did not include a clinical control group and therefore the specificity of the present findings with regard to chronic depression remains unclear. Second, about half of the patients participating in this study took antidepressant medication at the time when the study was conducted and it is unclear if this has influenced the results. Third, we did not assess cognitive reactivity following the sad mood induction and therefore it was not possible to test if increases in DMN connectivity were indeed related to activation of dysfunctional cognitive memory structures. Fourth, the majority of participants were female and it is possible that results would differ in a sample with more male participants. Fifth, we did not assess if participants suffered from neurological diseases other than those that are a contraindication for MRI (e.g., epilepsy). Finally, we did not apply False Discovery Rate (FDR) to control for multiple comparisons in this study. Instead, we used an uncorrected p-value of 0.01 and corrected at the cluster-level by empirically obtaining a cluster size threshold of 5 . By using clusterthresholding procedures we applied an empirically sound method to account for multiple comparisons at the cluster level.
The present study suggests that PCC connectivity with a number of DMN regions increases in cMDD patients but not in controls following a sad mood induction. This finding has potential clinical implications. Patients with cMDD might fail to exert cognitive control over memory retrieval processes once sad mood is triggered, as indicated by the decreased PCC - anterior prefrontal cortex connectivity in patients with cMDD. Non-patient controls seem to increase cognitive control processes possibly in an attempt to cope with the sad mood-state, as indicated by an increased PCC connectivity with the anterior prefrontal cortex. Increasing cognitive control in patients with depression either through neurostimulation [45] or through behavioral interventions might be a fruitful avenue in the treatment of cMDD. In conclusion, this study suggests that DMN connectivity in patients with cMDD is modulated by negative mood states.

\section{Role of Funding Source}

This work was support by Maastricht University and by the Academic Community Mental Health Centre (RIAGG) Maastricht.

\section{Acknowledgements}

We are grateful to our colleagues at Maastricht University who supported this study: Annie Hendriks and Annie Raven helped with patient recruitment; Danielle Jongen assisted with recruitment of matched controls; Thanks are also due to our colleagues at the Riagg Maastricht who helped to recruit patients and conducted clinical interviews for this study.

\section{References}

Richards, D., 2011]. Prevalence and clinical course of depression: a review. Clin. Psychol. Rev. 31 (7), 1117-1125.

Mulders, P.C., et al., 2015]. Resting-state functional connectivity in major depressive disorder: a review. Neurosci. Biobehav. Rev. 56, 330-344.

Hamilton, J.P., et al., 2015]. Depressive Rumination, the Default-Mode Network, and the Dark Matter of Clinical Neuroscience. Biol. Psychiatry 78 (4), 224-230.

Whitfield-Gabrieli, S., et al., 2011]. Associations and dissociations between default and self-reference networks in the human brain. Neuroimage 55 (1), 225-232.

Berman, M.G., et al., 2011]. Depression, rumination and the default network. Soc. Cogn. Affect Neurosci. 6 (5), 548-555.

Spreng, R.N., Mar, R.A., Kim, A.S., 2009]. The common neural basis of autobiographical memory, prospection, navigation, theory of mind, and the default mode: a quantitative meta-analysis. J. Cogn. Neurosci. 21 (3), 489-510.

Marchetti, I., et al., 2012]. The default mode network and recurrent depression: a neurobiological model of cognitive risk factors. Neuropsychol. Rev. 22 (3), 229-251.

Kaiser, R.H., et al., 2015]. Large-scale network dysfunction in major depressive disorder: a meta-analysis of resting-state functional connectivity. JAMA. Psychiatry.

Gudayol-Ferré, E., et al., 2015]. Changes IN brain connectivity related TO THE treatment OF depression measured THROUGH fMRI: a systematic review. Front. Hum. Neurosci., 9

Harrison, B.J., et al., 2008]. Modulation of Brain Resting-State Networks by Sad Mood Induction. PLoS ONE 3 (3), p. e1794.

Zamoscik, V., et al., 2014]. Increased involvement of the parahippocampal gyri in a sad mood predicts future depressive symptoms. Soc. Cogn. Affect Neurosci..

Greicius, M.D., et al., 2007]. Resting-state functional connectivity in major depression: abnormally increased contributions from subgenual cingulate cortex and thalamus. Biol. Psychiatry 62 (5), 429-437.

Renner, F., et al., 2016]. Schema therapy for chronic depression: results of a multiple single case series. J. Behav. Ther. Exp. Psychiatry 51, 66-73.

Martin, M., 1990]. On the induction of mood. Clin. Psychol. Rev. 10, 669-697.

Renner, F., et al., 2014]. Effects of a best-possible-self mental imagery exercise on mood and dysfunctional attitudes. Psychiatry Res. 215 (1), 105-110.

Werthmann, J., et al., 2014]. Looking at food in sad mood: do attention biases lead emotional eaters into overeating after a negative mood induction? Eat. Behav. 15 (2), 230-236.

Beck, A.T., Steer, R.A., Brown, G.K., 1996. Manual for the Beck Depression InventoryII, San Antonio, TX.

van der Does, A.J.W., 2002]. Handleiding: de Nederlandse versie van de Beck Depression Inventory, 2de Editie. [The Dutch version of the Beck Depression Inventory 2nd ed. Swets and Zeitlinger, Lisse, (b.v).

Weiskopf, N., et al., 2006]. Optimal EPI parameters for reduction of susceptibilityinduced BOLD sensitivity losses: a whole-brain analysis at $3 \mathrm{~T}$ and $1.5 \mathrm{~T}$. NeuroImage 33 (2), 493-504

Talairach, J., Tournoux, P., 1988]. Co-planar stereotaxic atlas of the human brain. Thieme, New York. 
Lancaster, J.L., et al., 2007]. Bias between MNI and Talairach coordinates analyzed using the ICBM-152 brain template. Hum. Brain Mapp. 28 (11), 1194-1205.

Greicius, M.D., et al., 2003]. Functional connectivity in the resting brain: a network analysis of the default mode hypothesis. Proc. Natl. Acad. Sci. USA 100 (1), 253-258.

Renner, F., et al., 2015]. Neural correlates of self-referential processing and implicit selfassociations in chronic depression. J. Affect Disord. 186, 40-47.

Northoff, G., et al., 2006]. Self-referential processing in our brain-A meta-analysis of imaging studies on the Self. Neuroimage 31 (1), 440-457.

Pannekoek, J.N., et al., 2014]. Aberrant resting-state functional connectivity in limbic and salience networks in treatment-naïve clinically depressed adolescents. J. Child Psychol. Psychiatry, (p. n/a-n/a).

Kim, B.-H., et al., 2015]. Altered resting-state functional connectivity in women with chronic fatigue syndrome. Psychiatry Res.: Neuroimaging 234 (3), 292-297.

Oertel-Knochel, V., et al., 2013]. Reduced functional connectivity and asymmetry of the planum temporale in patients with schizophrenia and first-degree relatives. Schizophr. Res 147 (2-3), 331-338.

van de Ven, V., et al., 2013]. Escitalopram decreases cross-regional functional connectivity within the default-mode network. PLoS One 8 (6), p. e68355.

Whitfield-Gabrieli, S., Ford, J.M., 2012]. Default mode network activity and connectivity in psychopathology. Annu Rev. Clin. Psychol. 8, 49-76.

Guo, W., et al., 2015]. Increased cerebellar-default-mode-network connectivity in drugnaive major depressive disorder at rest. Med. (Baltim.) 94 (9), p. e560.

Disner, S.G., et al., 2011]. Neural mechanisms of the cognitive model of depressionNature Reviews. Neuroscience. advance online publication.

Kaiser, R.H., et al., 2016]. Dynamic Resting-State Functional Connectivity in Major Depression. Neuropsychopharmacology 41 (7), 1822-1830.

A.E. Cavanna, M.R. Trimble. Precuneus: A Rev. its Funct. Anat. Behav. Correl., 129, 2006, pp. 564-583
Bluhm, R., et al., 2009]. Resting state default-mode network connectivity in early depression using a seed region-of-interest analysis: decreased connectivity with caudate nucleus. Psychiatry Clin. Neurosci. 63 (6), 754-761.

Zhu, X., et al., 2012]. Evidence of a Dissociation Pattern in Resting-State Default Mode Network Connectivity in First-Episode, Treatment-Naive Major Depression Patients. Biol. Psychiatry 71 (7), 611-617.

Williams, J.M., et al., 2007]. Autobiographical memory specificity and emotional disorder. Psychol. Bull. 133 (1), 122-148.

Fitzgerald, P.B., et al., 2008]. A meta-analytic study of changes in brain activation in depression. Hum. Brain Mapp. 29 (6), 683-695.

Canli, T., et al., 2004]. Brain activation to emotional words in depressed vs healthy subjects. Neuroreport 15 (17), 2585-2588.

Janata, P., 2005]. Brain networks that track musical structure. Ann. New Y. Acad. Sci. 1060 (1), 111-124.

Pehrs, C., et al., 2014]. How music alters a kiss: superior temporal gyrus controls fusiform-amygdalar effective connectivity. Soc. Cogn. Affect Neurosci. 9 (11), $1770-1778$.

Barredo, J., Öztekin, I., Badre, D., 2013]. Ventral fronto-temporal pathway supporting cognitive control of episodic memory retrieval. Cereb. Cortex.

Badre, D., Wagner, A.D., 2007]. Left ventrolateral prefrontal cortex and the cognitive control of memory. Neuropsychologia 45 (13), 2883-2901.

Gotlib, I.H., Joormann, J., 2010]. Cognition and depression: current status and future directions. Annu Rev. Clin. Psychol. 6, 285-312.

De Raedt, R., Koster, E.W., 2010]. Understanding vulnerability for depression from a cognitive neuroscience perspective: a reappraisal of attentional factors and a new conceptual framework. Cogn. Affect. Behav. Neurosci. 10 (1), 50-70.

Wolkenstein, L., Plewnia, C., 2013]. Amelioration of cognitive control in depression by transcranial direct current stimulation. Biol. Psychiatry 73 (7), 646-651. 